\title{
Pericarditis aguda en Puesto Avanzado de Combate (Afganistán): cuando un diagnóstico sencillo se convierte en complicado.
}

\author{
López Soberón E. ${ }^{1}$, Navarro Suay R. ${ }^{2}$, Álvarez Antón S. ${ }^{3}$, Méndez Fernández M. ${ }^{4}$ \\ Sanid. mil. 2013; 69 (4): 257-259; ISSN: 1887-8571
}

\section{RESUMEN}

Se presenta un caso de pericarditis aguda diagnosticado en el Puesto Avanzado de Combate «Ricketts» (Muqur, Afganistán), con las limitaciones logísticas y sanitarias presentes en Zona de Operaciones, que cursó con buena evolución hasta su curación.

Acute pericarditis in Combat Out Post (Afghanistan): when a simple diagnostic becomes complicate

SUMMARY: We present a case of acute pericarditis diagnosed in Combat Out Post «Ricketts» (Muqur, Afghanistan), showing the logistic and medical limitations that we can come across in-theatre, and that had a favourable evolution until complete recovery.

\section{INTRODUCCIÓN}

La pericarditis aguda es un síndrome clínico caracterizado por la inflamación del pericardio, que se puede afectar por agentes infecciosos, físicos, traumáticos, inflamatorios o, en forma secundaria, debido a procesos metabólicos o enfermedades generales ${ }^{1}$.

Se estima que la incidencia de este síndrome se aproxima al 5\% de las consultas por dolor torácico de origen no isquémico en los servicios de urgencias ${ }^{2}$.

El diagnóstico se realiza en base a la clínica, la exploración física y la electrocardiografía. La presencia de dolor torácico típico de pericarditis (retroesternal o precordial izquierdo, en ocasiones irradiado hacia el cuello y región dorsal izquierda, que suele aumentar en decúbito supino, con la tos, con la inspiración profunda y mejorar en la posición sentado), asociado o no a fiebre, la aparición en la auscultación de roce pericárdico (signo patognomónico pero cuya ausencia no excluye el diagnóstico) y la presencia de alteraciones electrocardiográficas evolutivas son característicos en esta enfermedad. La analítica de sangre con determinación de marcadores de daño miocárdico y el ecocardiograma en etapas precoces son útiles para la estratificación del riesgo. El tratamiento, en ausencia de factores de mal pronóstico, se puede realizar de forma ambulatoria, y se basa en el reposo absoluto y la utilización de AINEs a altas dosis ${ }^{1,2}$.

\footnotetext{
${ }^{1}$ Cap. Médico. Escuela Militar de Sanidad / Hospital Central de la Defensa «Gómez Ulla». Servicio de Cardiología. Madrid. España.

2 Cte. Médico. Hospital Central de la Defensa «Gómez Ulla». Servicio de Anestesiología y Reanimación Madrid. España.

3 Tcol. Médico. Hospital Central de la Defensa «Gómez Ulla». Servicio de Cardiología. Madrid. España.

${ }^{4}$ Col. Médico. Hospital Central de la Defensa «Gómez Ulla». Servicio de Cardiología. Madrid. España.

Dirección para correspondencia: Edurne López Soberón. Escuela Militar de Sanidad. Camino de los Ingenieros nº. CP: 27047 Madrid. helicobacter@gmail.com
}

Recibido: 17 de diciembre de 2012

Aceptado: 15 de abril de 2013
A continuación describimos el caso de una pericarditis aguda diagnosticada y tratada en un militar desplegado en una Base avanzada en Afganistán, con las limitaciones asistenciales y logístico-operativas que la situación conlleva.

\section{CASO CLÍNICO}

Se presenta el caso de un militar de 25 años (75 Kg, 174 $\mathrm{cm}$ ), sin antecedentes patológicos de interés, que acude al Puesto de Socorro del Puesto Avanzado de Combate «Ricketts» (Muqur, Afganistán) por referir dolor centro-torácico punzante, no irradiado, y continuo de 4 horas de evolución, que aumentaba con los movimientos respiratorios y empeoraba con el decúbito supino. No presentaba ni fiebre ni cuadro infeccioso previo.

En la exploración física no presentaba aumento de la presión venosa yugular, la auscultación pulmonar era anodina y en la auscultación cardiaca se objetivaron tonos cardiacos rítmicos, sin roce pericárdico. Se registraron las siguientes

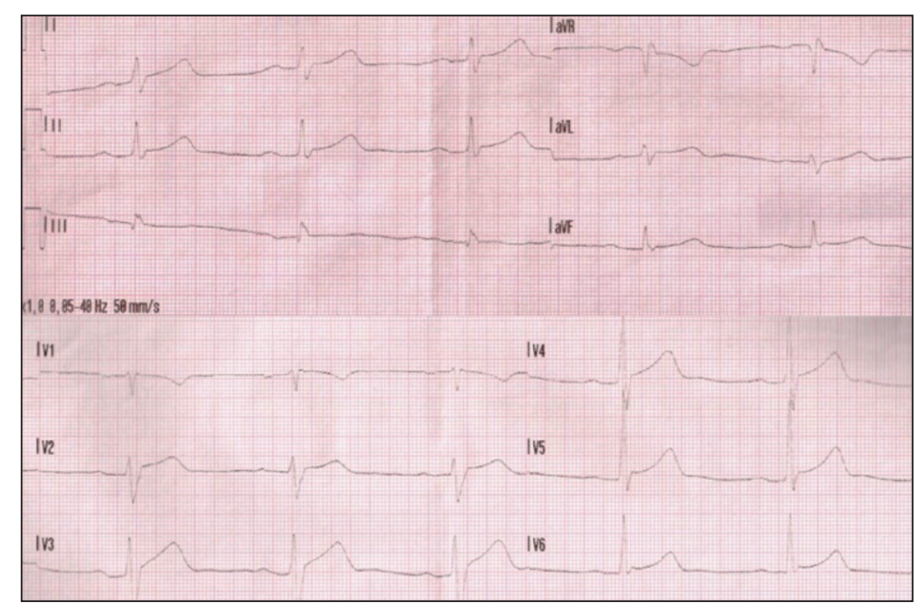

Figura 1. ECG de 12 derivaciones de la baja (imagen de los autores). 


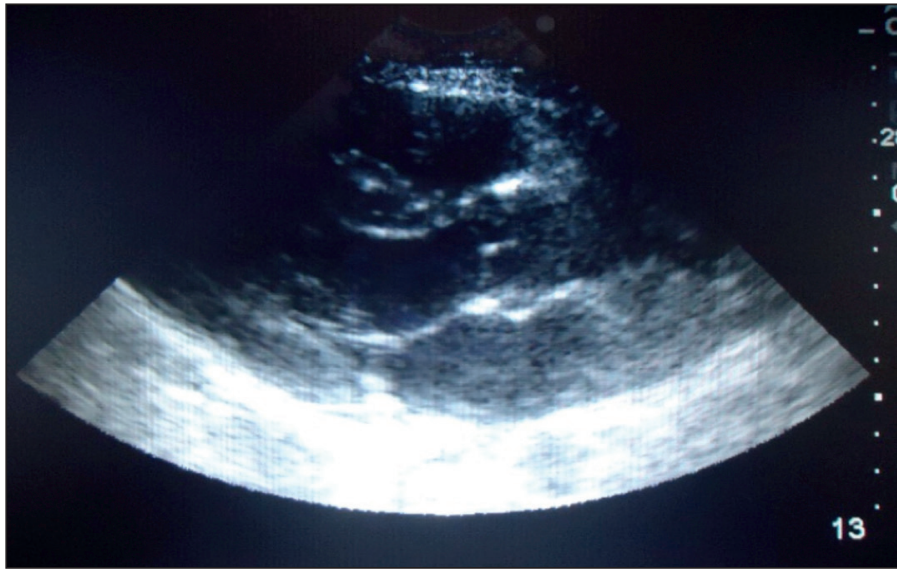

Figura 2. Ecocardiograma realizado sobre la baja. Proyección paraesternal eje largo (imagen de los autores).

constantes vitales: PANI: 115/73 mmHg, FC: 83 lpm y $\mathrm{SatO}_{2}$ basal: 98\%. El ECG mostraba un ascenso del punto $\mathrm{J}$ con ligera elevación del segmento ST en prácticamente todas las derivaciones (excepto aVR y V1) (Figura 1). Ante estos hallazgos, se realizó un ecocardiograma (Figura 2 y 3 ) en el que se constató una FEVI visual conservada con buena función del VD y ausencia de derrame pericárdico. Resultó inviable la realización de exámenes de laboratorio por no contar con dicha capacidad en el citado Puesto de Socorro. Sí se pudo realizar una consulta telemática con el Hospital Central de la Defensa «Gómez Ulla» (Madrid) a través de los medios de telemedicina disponibles en el Puesto de Socorro, con la intención de consultar el caso con un Oficial Médico diplomado en Cardiología para confirmar el diagnóstico y tomar decisiones conjuntas. Tras ello, y ante la ausencia de marcadores de mal pronóstico, se decidió mantener al enfermo en reposo absoluto instaurando tratamiento con ibuprofeno $600 \mathrm{mg} / 8 \mathrm{~h}$ v.o. decidiéndose además realizar electrocardiogramas y ecocardiogramas de control cada $48 \mathrm{~h}$, con el fin de valorar cambios precoces en la evolución. La evolución resultó satisfactoria remitiendo el dolor torácico al tercer día y consiguiéndose una recuperación total a los 7 días del inicio del cuadro.

\section{DISCUSIÓN}

La pericarditis aguda es una enfermedad frecuente en los Servicios de Urgencias, a cuyo diagnóstico se suele llegar sin demasiada dificultad en los hospitales de países occidentales.

La particularidad del caso presentado estriba en las limitaciones logísticas y sanitarias presentes en un Puesto Avanzado de Combate «Ricketts» (Muqur, en la Zona de Operaciones de Afganistán) donde se atendió a la baja. Este destacamento se encuentra aproximadamente a $25 \mathrm{Km}$ de Qala i Naw (donde se encuentra el Role 1 español) y a unos $200 \mathrm{Km}$ del Role 2 español de Herat. El Puesto Avanzado de Combate «Ricketts» cuenta con un Puesto de Socorro con capacidad sanitaria tipo Role 1, en el que hay de forma permanente un Oficial enfermero y un sanitario. A esta dotación se añade una Célula de Estabilización compuesta por un Oficial médico, un Oficial enfermero y un sanitario- conductor que se releva periódicamente. En dicha instalación sanitaria se tiene capacidad para el diagnóstico y tratamiento de enfermedades leves así como para una primera valoración, estabilización médica y puesta en estado de evacuación de las bajas de combate y de no combate que se produzcan. Dispone de medios de electromedicina para realizar un soporte vital avanzado (ventilador, oxígeno, desfibrilador, monitor de constantes vitales y capnógrafo). Se puede establecer una conexión de telemedicina tanto con el Hospital Central de la Defensa «Gómez Ulla» (capacidad Role 4), como con la Base de Qala i Naw (capacidad Role 1) y la Base de Herat (capacidad Role 2 «Enhanced») $)^{3}$. El equipo de telemedicina cuenta con un ecógrafo portátil con una sonda apta para realización de ecocardiografía ${ }^{4}$. No hay ningún medio que permita realizar analítica sanguínea. El Puesto Avanzado de Combate dispone de una zona de toma de helicópteros en caso de que fuera necesario realizar una aeroevacuación.

Tal distanciamiento kilométrico, sumado a la complicación existente para iniciar cualquier movimiento sobre el terreno (sea terrestre y/o aéreo) por el peligro existente en una zona de conflicto bélico activo, da una idea de las limitaciones existentes para evacuar cualquier baja. Por lo que a la hora de tomar decisiones, éstas, deben ser equilibradas con las premisas de riesgo y beneficio; además de valorar las repercusiones tácticas que una evacuación puede suponer al conjunto de la Operación. Sin que ello suponga demora alguna en la evacuación de cualquier baja que lo precise, ya que en Zona de Operaciones el Mando debe asegurar todos los medios y posibilidades de evacuación necesarias, sí se deben equilibrar muy concienzudamente todas las decisiones al respecto con el fin de conseguir que una baja «demorable» no precipite acontecimientos que supongan un riesgo o repercusión táctica en la Operación; y del mismo modo, que una baja «no demorable» no suponga la pérdida de una vida, si es que necesita ser evacuada de inmediato. Es ahí donde radica la «vital» importancia de mensurar adecuadamente la necesidad o no de una evacuación inmediata.

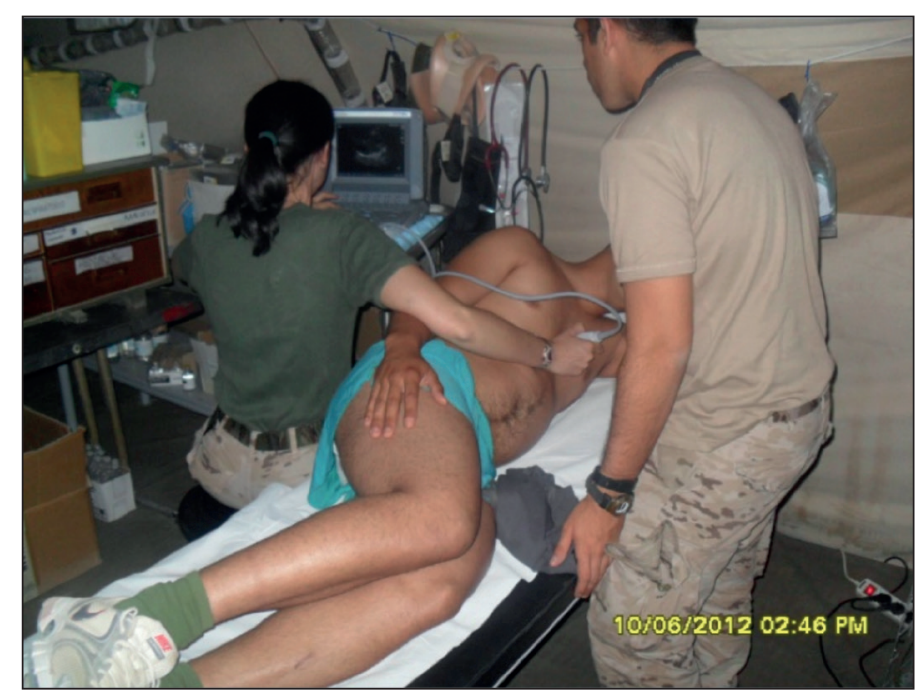

Figura 3. Capitán Médico realizando ecocardiograma en la enfermería del Puesto Avanzado de Combate "Ricketts" de Muqur (Afganistán) (imagen de los autores). 
La baja que nos ocupa en el caso presentaba dolor y un registro electrocardiográfico compatible con pericarditis. No se le pudo realizar una analítica sanguínea que estratificase el riesgo pero si un ecocardiograma, el cual fue normal; es por ello que se decidió tomar una actitud terapéutica conservadora posponiendo la evacuación en función de la posterior evolución, de la cual se hizo un seguimiento concienzudo. De este modo se consiguió minimizar el impacto táctico que sobre la Operación hubiera tenido una evacuación no urgente.

La telemedicina, sin ser definitiva para el diagnóstico del proceso patológico, el cual se pudo realizar con los medios disponibles, sí lo fue para confirmarlo por un especialista de la materia relacionada y para consensuar decisiones al respecto de forma compartida. Algo especialmente importante por lo expuesto anteriormente.

\section{CONCLUSIÓN}

De manera que, aunque la pericarditis aguda es una entidad frecuente que puede ser diagnosticada, estratificada y tratada eficazmente con los medios disponibles en los servicios de urgencias hospitalario, el mismo proceso patológico se torna complicado en situaciones donde las limitaciones diagnósticas y terapéuticas son escasas o inexistentes y además se ven limitadas las posibilidades de evacuación en tiem- po y forma. Es evidente que la capacidad de diagnóstico y tratamiento en el Puesto de Socorro de un Puesto Avanzado de Combate es limitada ${ }^{3}$; por ello, en nuestro caso, contar con oficiales médicos versados en patología general, disponer de determinados medios diagnósticos «clave» como la ecografía $^{4}$, conocer la logística sanitaria que nos apoya así como el fin táctico de la Operación en la que estamos inmersos y, por último, obtener la posibilidad de consultar mediante telemedicina ${ }^{5}$ con un Escalón Sanitario superior, hizo que se evitara la evacuación a retaguardia de la baja minimizando, con ello, el impacto táctico ${ }^{6}$ en la Unidad desplegada en Afganistán.

\section{BIBLIOGRAFÍA}

1. Freixa X. Evaluación, manejo y tratamiento de las pericarditis y miocarditis agudas en urgencias. Emergencias 2010; 22: 301-6.

2. Sagristá J. Pericarditis aguda. Med clin (Barc) 2004; 123 (13):505-8.

3. Navarro R, Bartolomé E, Jara I, Oreja A, González G. Capacidades y asistencia sanitaria realizada por el ROLE-2 español en la FSB de Herat (Afganistán) desde febrero a julio del 2007. Sanid Mil 2008; 64: 98-104.

4. Gorrea MA, Roel J, Hernández JC. Dotación de ecografía portátil en el módulo de Radiología de Campaña. Med Mil 1998; 54(1): 53-8.

5. Militares españoles en Afganistán disponen de equipos de telemedicina [citado 27 Nov 2011]. Disponible en: http://www.mde.es/gabinete/notasPrensa

6. Roberts J, McManus J, Harrison B. Uso de la ecografía para evitar un procedimiento innecesario en el entorno prehospitalario de combate: presentación de un caso. Prehospital emergency care 2006; 10:502-6. 